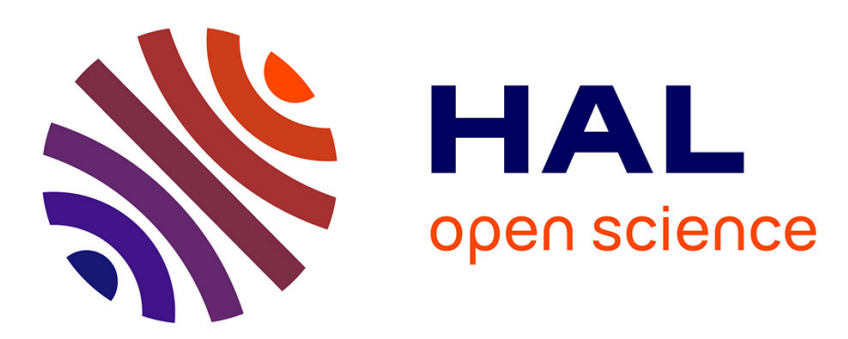

\title{
Essai d'évaluation de l'organisation et de la réorganisation de l'azote minéral du sol par la méthode des modèles compartimentaux. II. Cas d'un sol cultivé : relations sol-végétal
}

Robert Morel, Thérèse Lasnier

\section{To cite this version:}

Robert Morel, Thérèse Lasnier. Essai d'évaluation de l'organisation et de la réorganisation de l'azote minéral du sol par la méthode des modèles compartimentaux. II. Cas d'un sol cultivé: relations sol-végétal. Agronomie, 1984, 4 (3), pp.295-303. hal-00884637

\section{HAL Id: hal-00884637 \\ https://hal.science/hal-00884637}

Submitted on 1 Jan 1984

HAL is a multi-disciplinary open access archive for the deposit and dissemination of scientific research documents, whether they are published or not. The documents may come from teaching and research institutions in France or abroad, or from public or private research centers.
L'archive ouverte pluridisciplinaire HAL, est destinée au dépôt et à la diffusion de documents scientifiques de niveau recherche, publiés ou non, émanant des établissements d'enseignement et de recherche français ou étrangers, des laboratoires publics ou privés. 


\section{Essai d'évaluation de l'organisation et de la réorganisa- tion de l'azote minéral du sol par la méthode des modèles compartimentaux. II. Cas d'un sol cultivé : relations sol- végétal}

Robert MOREL

avec la collaboration technique de Thérèse LASNIER

I.N.R.A. - Chaire de Physico-Chimie et Science du Sol, I.N.A. Paris-Grignon F 78850 Thiverval-Grignon

Pour des sols cultivés, la recherche d'informations quantitatives sur la dynamique de l'azote et du carbone procède de la même démarche que celle proposée dans un précédent mémoire sur les sols sans culture. Trois hypothèses de travail, notamment, étaient sollicitées :

- Le classement de la matière organique du sol en deux catégories, l'essenticl de l'activité biologique se développant au sein de la fraction "labile».

- La constance, pour une situation donnée et pour une période suffisamment longue, de la valeur $\sigma$ du rapport $\mathrm{C} / \mathrm{N}$ du substrat organique en évolution, qui assure dans le sol un équilibre minéralisationréorganisation. Si C représente la quantité de carbone organique de ce substrat (fraction labile), ce dernier doit contenir une quantité d'azote $\mathrm{A}=\mathrm{C} / \sigma$ pour que s'établisse l'équilibre précité.

- Pour la durće de l'unité de temps choisie, l'azote minéral reçu par le sol et l'azote minéralisé participent à l'alimentation des organismes vivants et aux pertes selon leurs proportions respectives.

Afin de résoudrc le problème posé, ces hypothèses sont utilisćes dans le traitement d'un modèle compartimental dans lequel un compartiment, important au plan fonctionnel, est constitué par la fraction labile de la matière organique.

La proportion de l'azote minéralisé « réorganisée », de même que la proportion de l'azote minéral reçu par le sol qui est "organisée », sont définies par un coefficient $\omega$, unique ; $\omega=\frac{A-O}{A+M}$ où $O$ est la quantité d'azote contenue dans la fraction organique labile en évolution, $M$ représente la quantité d'azote minéral apportéc au sol.

La réorganisation et l'organisation de l'azote minćral dans le sol ne sont pas sans relation avec l'alimentation azotće de la plante ; un aspect compétitif associc les deux phénomènes: le coefficient u d'utilisation par le végétal de l'azote minéral reçu par le sol (pluies, engrais) est relié au coefficient de réorganisation $\omega$ dans l'expression : $u+\omega \leqq 1$. Le complément à 1 de la somme $u+\omega$ définit le « coefficient de pertes $\Pi$. Par aillcurs, la connaissance de $\omega$ permet de calculer le taux net annuel $\gamma$ de minéralisation de la matière organique du sol. Les concepts thćoriques présentés soulignent l'importance de $\sigma$ dans les processus de transferts de l'azote et du carbone dans le sol. Les résultats de la démarche proposée sont appliqués à différents traitements d'un dispositif parcellaire de longue duréc. Relevons, entre autres informations, l'importance du coefficient de pertes marquant l'absence de fertilisation phosphatée et la valeur élevée du cocfficient d'utilisation u, précédemment défini, dans les parcelles ne recevant pas de fumure azotée.

Mots clés additionnels : Rapport $C / N$, transferts d'azote, minéralisation de l'azote organique, coefficient d'utilisation, pertes d'azote.

Attempt to estimate the organization and reorganization of soil nitrogen using the compartment models method. II. Case of cultivated soil ; soil-vegetation relations.

A quantitative study of the nitrogen and carbon relationships in cultivated soils has been made following the same procedures as in our preceding paper concerning uncultivated soils. Three working hypotheses were put forward:

1. Classification of the organic matter into two categories, with the essential biological activity developing in the labile fraction.

2. When a given situation continues for a sufficiently long period, there is a constant value $\sigma$ for the $\mathrm{C} / \mathrm{N}$ ratio of the developing organic substances for a mincralization-reorganization equilibrium in the soil. If $\mathrm{C}$ 
represents the amount of organic carbon in the labile fraction of the substratc, a specific amount of nitrogen $\mathrm{A}=\mathrm{C} / \boldsymbol{\sigma}$ must be present in order to establish the forementioned equilibrium.

3. During a given period of time, the mineral nitrogen received by the soil, and the nitrogen mineralized in the soil, supply living organisms and provide for nitrogen losses according to their respective proportions. In order to resolve the proposed problem, these hypotheses were applied to a compartment model, in which the compartment important to overall functioning is the labile fraction of the organic matter.

The ratio of ' reorganized ' mineralized nitrogen and the ratio of mineral nitrogen received by the soil which is 'organized', are defined by the samc cocfficient $\omega=\frac{A-O}{A+M}, O$ is the amount of nitrogen in the developing labile organic fraction; $M$ represents the amount of mineral nitrogen given to the soil.

The reorganization and the organization of the mineral nitrogen in the soil are related to the plant's nitrogen supply: the two phenomena are in competition. The plant utilization coefficient, u, of mineral nitrogen received by the soil (rain, fertilizer) is related to the rcorganization coefficient $\omega$ by the equation : $u+\omega \leqq 1$. The difference between 1 and the sum of $u+\omega$ defines the loss coefficient $\Pi$. Thus, knowing the value of $\omega$, the net yearly mineralization rate $\gamma$ of the organic soil component can be calculated.

The theoretical concepts presented underline the importance of $\sigma$ in nitrogen and carbon transfer procedures in the soil.

The results of the proposed procedure were applied, using different treatments, on a set of long-term plots. It is important to note that the absence of phosphate fertilization was marked by a high loss coefficient and that the utilization cocfficient, previously defined, was high in plots which did not receive nitrogen fertilizer.

Additional key words : $C / N$ ratio, nitrogen transfers in soil, mineralization of organic nitrogen, utilization coefficient, soil nitrogen losses.

\section{TABLEAU 1}

Liste et signification des symboles utilisés List and meaning of symbols

- Compartiment I : fraction labile de la matic̀rc organique du sol, labile organic matter fraction of the soil.

- Compartiment II : fractions organiques stables du sol, stable organic fractions of the soil.

- Compartiment III : parties aériennes exportées, aerial plant parts removed.

A = Azote que doivent contenir les substances organiques du compartiment I pour le maintien de l'équilibre minéralisation-réorganisation, nitrogen needed in organic matter of compartment I to maintain the mineralization-reorganization equilibrium.

$\mathrm{C}=$ Carbone organique entrant dans le compartiment $\mathrm{I}$, carbon entering compartment $\mathrm{I}$.

$\mathrm{C}_{1}=$ carbone organique reçu par le sol, organic carbon received by soil.

$\mathrm{C}_{2}=$ carbone organique provenant du compartiment II, organic carbon from compartment II.

$\mathrm{C}_{3}=$ carbone organique provenant des racines, organic carbon from roots.

$\mathbf{M}=$ Azote minéral reçu par le sol, mineral nitrogen received by soil.

$\mathbf{x}=$ fraction de $\mathbf{M}$ alimentant les parties aériennes, fraction going to aerial parts.

$\mathrm{a}_{1}=$ fraction de $M$ fixée dans les racines, fraction fixed in roots.

$\mathrm{a}_{2}=$ fraction de $\mathrm{M}$ organisée par les microorganismes, fraction organized by microorganisms.

$\mathrm{b}=$ fraction de $\mathrm{M}$ évacuée dans les pertes, fraction lost.

$\mathrm{m}=$ azote minéralisé provenant du compartiment $\mathrm{I}$, mineralized nitrogen from compartment $\mathrm{l}$.

$\mathrm{Y}=$ fraction de $\mathrm{m}$ alimentant les parties aćricnnes, fraction going to aerial parts.

$\mathrm{d}_{1}=$ fraction de $\mathrm{m}$ fixée dans les racines, fraction fixed in roots.

$\mathrm{d}_{2}=$ fraction de $\mathrm{m}$ réorganisée par les microorganismes, fraction reorganized by microorganisms.

$\mathrm{e}=$ fraction de $\mathrm{m}$ évacuée dans les pertes, fraction lost.

$\mathrm{N}_{\mathrm{r}}=$ Azote contenu dans les racines, nitrogen in roots.

$\mathrm{O}=$ Azote organique entrant dans le compartiment I, organic nitrogen entering compartment $\mathrm{I}$.

$\mathrm{O}_{1}=$ azote organique reçu par le sol, organic nitrogen received by soil.

$\mathrm{O}_{2}=$ azote organique provenant du compartiment II, organic nitrogen from compartment $\mathrm{II}$.

$\mathrm{R}=$ Azote des organes aériens récoltés et exportés, nitrogen in aerial parts harvested and removed.

$\mathrm{u}=$ Coefficient d'utilisation par le végétal, à la récolte, de l'azote minćral (engrais + pluies) reçu par le sol, coefficient of utilization by the plant, at harvest, of mineral nitrogen (fertilizer input + rain) received by the soil.

$\gamma=$ Taux net annuel de minéralisation de l'azote organique du sol, annual mineralization rate of organic nitrogen in the soil.

$\sigma=$ Valeur du rapport $\mathrm{C} / \mathrm{N}$ des substances organiques en évolution dans le compartiment I pour laquelle il ne se produit ni minéralisation, ni réorganisation nettes, value of the $C / N$ ratio for organic matter in compartment $I$ at which there is no net mineralization or reorganisation.

$\omega=$ Coefficient de réorganisation de $\mathrm{m}$ dans la biomasse du compartiment $\mathrm{I}$, coefficient of reorganization of $m$ in the biomass of compartment $I$. $\omega_{1}=$ dans celle des racines, coefficient of reorganization of $m$ in the biomass of the roots.

$\omega_{2}=$ dans celle des microorganismes, coefficient of reorganization of $m$ in the biomass of microorganisms. 


\section{INTRODUCTION}

Nous avions, dans un précédent mémoire, proposé une démarche susceptible de traiter quantitativement le problème des transferts d'azote dans un sol sans culture (MOREL, 1981a).

Dans le présent document, nous étendons la démarche au cas d'un sol cultivé ; nous nous sommes fixé comme objectif celui d'aborder la question avec la seule disposition des données habituelles aisément rassemblées dans le cadre de l'exploitation agricole : masses végétales exportées, apports organiques et minéraux au sol, teneurs en carbone et en azote du sol et des récoltes. Cette option répond, en particulier, au souhait d'apporter des informations quantitatives aisément accessibles au dossier ouvert sur les aspects souvent préoccupants de l'entretien organique des terres cultivées.

Les données utilisćes se réfèrent à une masse importante de sol (masse correspondant à la couche arable évaluée sur une superficie de 1 hectare). L'unité de temps choisie correspond à un cycle biologique annuel moyen, tenant compte de l'ensemble des rotations culturales établies sur la parcelle étudiée ; ainsi la démarche suivie repose $t$-elle sur des observations réalisées à moyen ou à long terme.

De même que pour la $1^{\text {re }}$ partie, la question est abordée par la voie d'un modèle compartimental dont l'étude requiert les hypothèses de travail que nous rappelons ciaprès :

1) Le critère distinctif des compartiments concernant le sol repose sur le degré de vulnérabilité de la matière organique du sol à l'activité des microorganismes; ce critère conduit à distinguer 2 compartiments : l'un, le compartiment I, comprenant la fraction «labile » de la matière organique, au sein de laquelle se développent intensément les processus biochimiques responsables de la minéralisation et de l'humification; l'autre, le compartiment II, rassemblant les substances organiques dites « stables » et de ce fait peu réactives. En raison de cette faible réactivité, la masse organique de ce compartiment est largement supérieure à celle du compartiment $\mathrm{I}$.

2) Le compartiment I est supposé en «état stationnaire », les flux entrants égalant les flux sortants; cette hypothèse est, dans ce mémoire, appliquée à l'azote, quelle que soit la forme de l'élément mise en jeu.

3) La valeur du rapport $\mathrm{C} / \mathrm{N}$ de l'ensemble des substrats organiques en évolution dans le compartiment I est choisie comme élément de référence prévisionnel quant à la destinée biochimique de l'azote : au-delà d'une certaine valeur $\sigma$ de ce rapport, l'azote minéralisé dans le compartiment se trouve totalement engagé dans un processus de réorganisation microbienne; en deçà de cette valcur, l'azote des substrats est non sculement en quantité suffisante pour assurer le développement microbien, mais il en subsiste un excès qui apparaît sous forme minérale (minéralisation primaire).

Si C représente la quantité de carbone contenue dans la masse des substances organiques mises en jeu dans le compartiment I au cours de l'unité de temps choisie, la valeur $\mathrm{A}=\mathrm{C} / \sigma$ exprime la quantité d'azote que doivent contenir ces substances pour qu'il ne se produise ni minéralisation, ni réorganisation nettes (équilibre minéralisationréorganisation); lorsque la quantité d'azote des substrats est inférieure à $\mathrm{A}$, la masse microbienne prélève dans son environnement le supplément qui lui est nécessaire.

Dans le sol, $\sigma$ est sans aucun doute fonction des proportions et de l'activité des différentes espèces microbiennes qui occupent le site concerné, proportions et activité ellesmêmes variables avec les qualités d'hébergement de ce site pour la microflore à l'instant considéré. Dans notre démarche, nous formons l'hypothèse que, pour une parcelle cultivée située dans un complexe pédo-climatique et cultural donné, $\sigma$ demeure constant ou très peu variable pour une masse importante de sol et au cours de périodes successives suffisamment longues.

4) Pour la durée de l'unité de temps choisie, l'azote minéral reçu par le sol d'une part, l'azote minéralisé d'autre part, participent à l'alimentation des organismes vivants et aux pertes selon leurs proportions respectives. Cette hypothèse est l'homologue de celle de FRIED \& DEAN (1952), ici généralisée à l'ensemble des transferts d'azote minéral, pertes comprises, et étendue à la durée de l'unité de temps.

Les hypothèses présentées permettent d'accéder, au plan quantitatif, aux phénomènes de réorganisation de l'azote minéral dans le sol. Corrélativement, la connaissance du coefficient de réorganisation ainsi calculé donne accès à une estimation du taux net annuel de minéralisation de l'azote organique du sol, considéré dans son ensemble (MOREL, 1981).

Par ailleurs, l'alimentation azotée des microorganismes et celle du végétal sont sur le même site de sol dans une situation concurrentielle. Le système compartimental proposé offre la possibilité, pour la durée de l'unité de temps choisie et pour une masse de sol suffisamment importante, d'établir une relation entre l'ampleur des processus de réorganisation et le coefficient d'utilisation pour la plante de l'azote minéral reçu par le sol.

\section{II. ÉTUDE DU SYSTÈME COMPARTIMENTAL}

\section{A. Description du système}

Le système proposé associe les compartiments suivants représentés dans la figure 1 pour ce qui concerne l'azote :

\section{Un compartiment $l$,}

constitué par la fraction labile de la matière organique du sol, et que caractérise une activité biochimique intense. Pour la clarté de la présentation, ce compartiment est divisé en 2 sous-compartiments :

a) le sous-compartiment $I_{a}$, quantitativement le plus important et qui comprend:

- les apports organiques au sol ;

- les masses racinaires et les résidus aériens non récoltés (chaumes de céréales par exemple);

- les composés organiques en voie de minéralisation issus du compartiment II, cela si le sol se trouve dans la situation d'un abaissement du taux de matière organique ; dans le cas contraire, c'est la masse organique du compartiment $I_{a}$ qui perd une fraction de sa substance au bénéfice du compartiment II. Rappelons que, dans le $1^{\text {er }}$ cas, nous postulons que les composés organiques du compartiment II en cours de minéralisation transitent obligatoirement par le compartiment $\mathrm{I}_{\mathrm{a}}$ (MOREL, 1981) ;

- la fraction de la micropopulation qui, au cours de l'unité de temps, n'a pas participé aux processus de réorganisation ou d'organisation. Cette masse microbienne trouve l'azote qui lui est nécessaire dans les substrats organiques précités. 


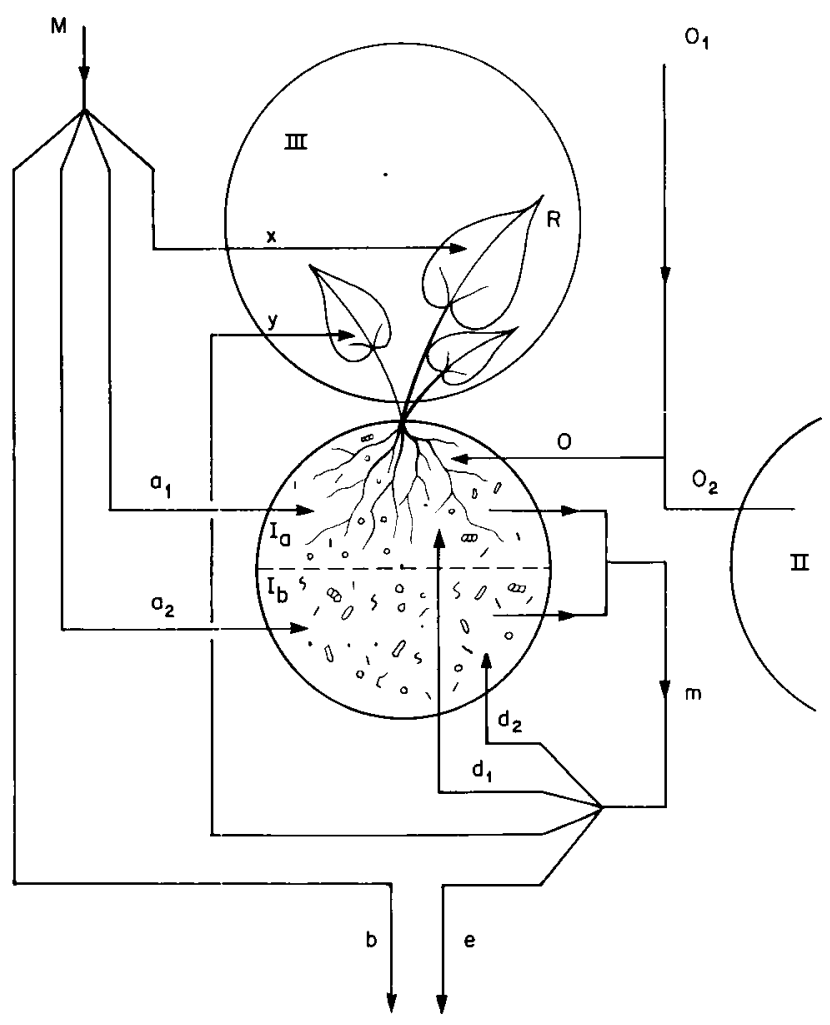

Figure 1

Modèle compartirnental concernant l'azote (voir tabl. 1, la signification des symboles utilisés).

Nitrogen compartmental model (see table 1 for the meaning of the symbols).

b) le sous-compartiment $I_{b}$, contenant la seule fraction des micro-organismes concernée par la réorganisation de l'azote minéralisé ou l'organisation de l'azote minéral apporté au sol; ce sous-compartiment $\mathrm{I}_{\mathrm{b}}$ n'existe pas si le recyclage de l'azote est nul. Soulignons aussi que cette division en 2 fractions microbiennes n'implique aucune référence à une spécificité fonctionnelle.

\section{Un compartiment $I I$,}

rassemblant toutes les fractions organiques stables du sol, comprenant essentiellement les substances humiques engagées ou non dans les liaisons avec la partic minérale du sol.

\section{Un compartiment III,}

comprenant les parties aériennes exportées; les parties aćriennes récoltées puis enfouies, comme les pailles de céréales, sont considérées comme des apports organiques au compartiment $\mathrm{I}_{\mathrm{a}}$.

\section{B. Transferts dans le système compartimental}

Nous nous placerons dans le cas où, les apports extéricurs demeurant constants, les taux de carbone et d'azote organiques du sol diminuent au cours de l'unitć de temps choisie. Les entrées dans le compartiment I sont alors les suivantes: sol),

- apports d'azote minćral (minćralisć ou reçu par le $\left(C_{1}\right)$,

- apports ex:érieurs organiques azotés $\left(\mathrm{O}_{1}\right)$ et carbonés

- apports carbonés par les racines $\left(\mathrm{C}_{3}\right)$,

- apports organiques azotés $\left(\mathrm{O}_{2}\right)$ et carbonés $\left(\mathrm{C}_{2}\right)$ en provenance du compartiment II. Rappelons ici la remarque que nous avons faite pour un sol sans culture : dans le cas étudié, qui postule un abaissement de la teneur du sol en matière organique, les pertes organiques du compartiment II participent dans leur intégralité à l'activité réactionnelle du compartiment I. Aussi considèrerons nous la diminution observée des quantités de carbone $\left(\mathrm{C}_{2}\right)$ et d'azote $\left(\mathrm{O}_{2}\right)$ comme des entrécs dans le compartiment I.

En définitive, au cours de l'unité de tetmps, les entrées dans le compartiment I sont :

pour l'azote organique $\mathrm{O}=\mathrm{O}_{1}+\mathrm{O}_{2}$, pour le carbone organique $\mathrm{C}=\mathrm{C}_{1}+\mathrm{C}_{2}+\mathrm{C}_{3}$.

Les sorties, quant à elles, sont représentées par les composés minéraux :

$\mathrm{CO}_{2}$ pour le carbone,

$\mathrm{NH}_{4}^{+}$et $\mathrm{NO}_{3}^{-}$pour l'azote.

La quantité $\mathrm{M}$ d'azote minéral apportée au sol, de même que la quantité $m$ d'azote minéral issue des processus de minéralisation, sc répartissent respectivement (fig. 1) :

- dans les organes aériens récoltés et exportés $(R)$,

- dans les racines $\left(\mathrm{N}_{\mathrm{r}}\right)$,

- dans la fraction de la biomasse microbienne concernée par la réorganisation de l'azote minéralisé ct l'organisation de l'azote minéral apporté au sol,

- dans les pertes.

\section{Relations quantitatives}

En considération des données précédentes, 3 groupes de relations sont établies dont chacun des termes se rapporte, ainsi que nous l'avons déjà précisé, aux quantités mises en jeu sur 1 ha de couche arable et pour l'année moyenne d'unc rotation culturalc.

1) Un $1^{\text {er }}$ groupe de relations exprime des bilans particls de transferts (fig. 1).

$$
\begin{gathered}
M=x+a_{1}+a_{2}+b \\
R=x+y \\
N_{r}=a_{1}+d_{1} \\
M+O=R+b+c
\end{gathered}
$$

L'équation (4) traduit le fait que le compartiment I fonctionne en régime stationnaire, les flux d'azote entrant et sortant étant égaux. Cet état apparaît normal dans un sol en équilibre azoté. Dans le cas présenté ici (abaissement de la teneur en matière organique), la grandeur $\mathrm{O}_{2}$ diminue très lentement ; cette diminution s'inscrit dans les entrées du compartiment I, alors que les sorties de ce compartiment correspondent aux entrées relatives à l'unité de temps précédente, légèrement plus élevées. La différence entre les 2 flux porte sur des quantités d'azote très faibles en regard des grandeurs $\mathrm{M}$ et $\mathrm{O}_{1}$; le caractère stationnaire, suggéré pour le fonctionnement du compartiment I, demeure, semble-t-il, une proposition acceptable.

2) Le $2^{e}$ groupe de relations traduit le comportement de la masse microbienne quant à ses besoins en carbone et en azotc. Si le compartiment I (substances organiques labiles) contient dans sa masse organique une quantité d'azote supéricure à $\mathrm{A}=\mathrm{C} / \sigma$, les processus de réorganisation (recyclage) ne sont pas nécessaires. Ils le sont dans le cas contrairc.

La quantité d'azote organique mise en jeu dans ce compartiment au cours de l'unité de temps, se compose 
quant à elle :

- de la masse $\mathrm{O}$ déjà définie,

- de la quantité $\mathrm{N}_{\mathrm{r}}$ d'azote des racines, formée au cours de l'unité de temps précédente : nous supposerons que cette quantité $\mathrm{N}_{\mathrm{r}}$ est entièrement libéréc au cours de l'unité de temps dans le compartiment I.

Deux cas sont alors possibles :

$-1^{\text {er }}$ cas : A est supérieur à la somme $\left(\mathrm{O}+\mathrm{N}_{\mathrm{r}}\right)$, c'est-àdire $A-\left(\mathrm{O}+\mathrm{N}_{\mathrm{r}}\right)>0$. Un apport d'azote minéral se révèle nécessaire pour satisfaire à l'alimentation azotée $\mathrm{dc}$ la masse microbienne, apport égal à $\mathrm{A}-\left(\mathrm{O}+\mathrm{N}_{\mathrm{r}}\right)$. $\mathrm{Ce}$ complément trouve son origine, d'une part, dans l'azote minéral reçu par le sol, d'autre part, dans l'azote issu des processus de minéralisation: nous pouvons écrire (fig. 1) :

$$
A-\left(\mathrm{O}+\mathrm{N}_{\mathrm{r}}\right)=\mathrm{a}_{2}+\mathrm{d}_{2}
$$

$2^{\mathrm{e}}$ cas: A est inférieur à $\left(\mathrm{O}+\mathrm{N}_{\mathrm{r}}\right)$ ou bien $A-\left(\mathrm{O}+\mathrm{N}_{\mathrm{r}}\right)<0$.

Le substrat organique du compartiment I est suffisamment pourvu en azote pour assurer l'évolution biochimique de la quantité de carbone organique contenuc dans ce substrat. L'excès d'azote, en quantité égale à $\left(\mathrm{O}+\mathrm{N}_{\mathrm{r}}\right)-\mathrm{A}$, apparaît sous forme minérale après minéralisation.

3) Enfin, un $3^{e}$ groupe d'expressions procède de l'établissement de relations homologues entre toutes les quantités se rapportant aux entrées et sorties compartimentales.

Ces relations relèvent de la proposition suivante :

Considérées pour l'unité de temps prise dans son ensemble, les quantités d'azote minéral absorbées par les organismes vivants d'une part, éliminées dans les pertes d'autre part sont, quant à leurs origines respectives, proportionnelles aux quantités $M$ d'azote minéral apportées et d'azote minéralisé $\mathrm{m}$ mises en jeu au cours de l'unité de temps.

Cette proposition traduit l'hypothèse que les microorganismes et les racines ne font aucune distinction entre l'azote minéral apporté et l'azote minéralisé ; il en est de même pour les pertes (FRIED \& DEAN, 1952).

L'hypothèse ainsi proposée conduit à la suite de rapports :

$$
\frac{\mathrm{x}}{\mathrm{y}}=\frac{\mathrm{a}_{1}}{\mathrm{~d}_{1}}=\frac{\mathrm{a}_{2}}{\mathrm{~d}_{2}}=\frac{\mathrm{b}}{\mathrm{e}}=\frac{\mathrm{M}}{\mathrm{m}} .
$$

\section{Résolution du système d'équations - Coefficient de réorganisation}

La résolution du système d'équations établi (relations 1 à 6) donne les valeurs des différents transferts du modèle compartimental, pour les 2 cas possibles de réorganisation et de non-réorganisation apparentes de l'azote (tabl. 2).

Les coefficients de réorganisation $\omega_{1}$ et $\omega_{2}$ de l'azote minéralisé $m$ dans la biomasse des sous-compartiments $I_{a}$ et $\mathrm{I}_{\mathrm{b}}$ ont pour expressions :

$$
\omega_{1}=\frac{\mathrm{d}_{1}}{\mathrm{~m}} \text { (racines) et } \omega_{2}=\frac{\mathrm{d}_{2}}{\mathrm{~m}} \text { (micro-organismes) }
$$

Le coefficient de réorganisation $\omega$ de l'azote minéralisé $m$ dans la biomasse du compartiment I se définit par la somme $\omega=\omega_{1}+\omega_{2}$ c'est-à-dire $\frac{d_{1}+d_{2}}{m}$ ou, en remplaçant $d_{1}$ et $d_{2}$ par leurs valeurs :

$$
\omega=\frac{A-O}{A+M}
$$

\begin{tabular}{|c|c|c|}
\hline & Cas de réorganisation & 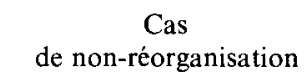 \\
\hline \multirow[b]{2}{*}{$x$} & MR & MR \\
\hline & $\overline{A+M}$ & $\overline{O+N_{r}+M}$ \\
\hline $\mathrm{y}$ & $\mathrm{AR}$ & $\left(\mathrm{O}+\mathrm{N}_{\mathrm{r}}\right) \mathrm{R}$ \\
\hline$y$ & $\overline{A+M}$ & $\overline{O+N_{r}+M}$ \\
\hline$a_{1}$ & $\frac{M N_{r}}{A+M}$ & $\frac{\mathrm{MN}_{\mathrm{r}}}{\mathrm{O}+\mathrm{N}_{\mathrm{r}}+\mathbf{M}}$ \\
\hline$a_{2}$ & $\frac{M\left(A-O-N_{r}\right)}{A+M}$ & zéro \\
\hline$d_{1}$ & $\frac{\mathrm{AN}_{\mathrm{r}}}{\mathrm{A}+\mathrm{M}}$ & $\frac{\left(\mathrm{O}+\mathrm{N}_{\mathrm{r}}\right) \mathrm{N}_{\mathrm{r}}}{\mathrm{O}+\mathrm{N}_{\mathrm{r}}+\mathrm{M}}$ \\
\hline $\mathrm{d}_{2}$ & $\frac{A\left(A-O-N_{r}\right)}{A+M}$ & zéro \\
\hline$b$ & $\mathrm{M}(\mathrm{M}+\mathrm{O}-\mathrm{R})$ & $\mathrm{M}(\mathrm{M}+\mathrm{O}-\mathrm{R})$ \\
\hline D & $A+M$ & $\overline{O+N_{r}+M}$ \\
\hline $\mathrm{e}$ & $\underline{A(M+O-R)}$ & $\left(\mathrm{O}+\mathrm{N}_{\mathrm{r}}\right)(\mathrm{M}+\mathrm{O}-\mathrm{R}$ \\
\hline e & $A+M$ & $\mathrm{O}+\mathrm{N}_{\mathbf{r}}+\mathrm{M}$ \\
\hline$\omega_{1}$ & $\frac{N_{r}}{A+M}$ & $\frac{N_{r}}{O+N_{r}+M}$ \\
\hline$\omega_{2}$ & $\frac{A-O-N_{r}}{A+M}$ & \\
\hline $\mathrm{m}$ & A & $\mathrm{O}+\mathrm{N}_{\mathrm{r}}$ \\
\hline
\end{tabular}

TABLEAU 2

Expressions des différents transferts compartimentaux. Expressions for different compartmental transfers.

L'expression de $\omega$ est aussi celle du « coefficient d'organisation " de l'azote minéral $\mathrm{M}$ dans les racines et les microorganismes du compartiment, somme des 2 valeurs partielles

$$
\omega_{1}=\frac{a_{1}}{\bar{M}} \quad \text { et } \quad \omega_{2}=\frac{a_{2}}{M} .
$$

Ce coefficient marque la contribution des apports minéraux azotés à l'élaboration de la matière organique du sol.

Le coefficient $\omega$, défini par la relation (7), est fonction de la quantité $M$ d'azote minéral d'une part, des quantités de carbone $\mathrm{C}$ et d'azote organiques entrant dans le compartiment I d'autre part (termes $\mathrm{O}$ et $\mathrm{A}=\mathrm{C} / \sigma$ ). Il est difficile d'analyser l'influence de chacun de ces facteurs sur le coefficient $\omega$ : un changement des quantités $C$ (apports organiques au sol) ou $\mathbf{M}$ entraîne une variation de la production végétale et, de ce fait, une modification des grandeurs $\mathrm{A}$ et $\mathrm{O}$.

\section{E. Relations entre le sol et le végétal}

Au plan nutritif, les parties aériennes des plantes se comportent comme des organes concurrentiels des masses vivantes souterraines, micro-organismes et racines: ces 2 ensembles vivants, aérien et souterrain, utilisent en effet pour leurs besoins alimentaires le même environnement, le sol.

Par ailleurs, afin de répondre à certaines préoccupations posées par l'alimentation azotée du végétal, l'attention s'est portée depuis de nombreuses années sur la notion de coefficient d'utilisation, ce dernier exprimant la fraction de 
l'azote minéral apporté au sol (engrais) exportée dans la récolte. Naguère estimé par comparaison de résultats expérimentaux obtenus sur parcelles cultivées avec ou sans incorporation d'azote minéral au sol, le coefficient d'utilisation est actuellement déterminé par voie isotopique (RÉMY \& VIAUX, 1980 ; BONIFACE, 1981). Une étude comparative des 2 méthodes a été récemment développée sur le sujet (LINDEMANN et al., 1981).

Pour les conditions habituelles de culture, le calcul du coefficient d'utilisation néglige généralement la quantité d'azote, relativement faible, apportée au sol par les pluies. Dans les applications étudiées dans ce mémoire, le coefficient d'utilisation se rapporte à l'azote reçu par le sol (engrais, pluies), les pluies représentant, pour certains des exemples étudies, la seule source d'azote minéral.

Cet ensemble de considérations engage à rechercher des relations d'interdépendance entre les différents transferts d'azote relatifs au complexe sol-plante.

Dans le système compartimental étudié, le cocfficient d'utilisation $u$ s'exprime par le rapport $u=\frac{X}{M}$ (fig. 1), ou encore $\mathrm{u}=\frac{\mathrm{R}}{\mathrm{A}+\mathrm{M}}$ (tabl. 2).

De même que pour le coefficient $\omega$, il est difficile de saisir l'incidence sur u des variations de $\mathrm{A}$ ou de $\mathbf{M}$ : celles-ci modifient l'importance de la production végétale et, en conséquence, les valeurs de $\mathrm{R}$ et de $\mathrm{A}$.

Le coefficient d'utilisation u peut être sans difficulté relié aux grandeurs $\sigma$ et $\omega$, comme l'indiquent les relations suivantes :

$$
\begin{aligned}
u & =\frac{\sigma R}{C+\sigma M} \\
u & =(1-\omega) \frac{R}{M+O} \\
\sigma & =\frac{u C}{R-u M} \\
\omega & =1-u \frac{(M+O)}{R} .
\end{aligned}
$$

L'aspect compétitif qui, au plan de l'alimentation azotée, marque les comportements relatifs des 2 ensembles vivants, aérien et souterrain, s'exprime dans une relation simple. La somme $u+\omega$ a en effet pour valeur :

$$
\mathrm{u}+\omega=1-\frac{(\mathrm{M}+\mathrm{O})-\mathrm{R}}{\mathrm{A}+\mathrm{M}} .
$$

Le terme $\frac{(M+O)-R}{A+M}$ est aussi égal à $\frac{b+e}{M+m}, A$ étant équivalent à m (tabl. 2). Or, le rapport $\frac{\mathrm{b}+\mathrm{e}}{\mathrm{M}+\mathrm{m}}$ indique la fraction de $M$ ou de $m$ qui disparait dans les pertes; en effet (fig. 1) :

$$
\frac{b}{M}=\frac{e}{m}=\frac{b+e}{M+m} .
$$

Désignons cette fraction par II qui représente le « coefficient de pertes". Nous pouvons alors écrire :

$$
u+\omega+\Pi=1
$$

u et $\omega$, complémentaires à 1 si les pertes sont nulles, satisfont à la relation générale :

$$
\mathbf{u}+\omega \leqslant 1 .
$$

\section{Remarque}

La relation (6) $\mathrm{x} / \mathrm{y}=\mathrm{M} / \mathrm{m}$ peut s'écrire :

$$
\mathrm{u}=\frac{\mathrm{x}}{\mathrm{M}}=\frac{\mathrm{y}}{\mathrm{m}}=\frac{\mathrm{x}+\mathrm{y}}{\mathrm{M}+\mathrm{m}}=\frac{\mathrm{R}}{\mathrm{M}+\mathrm{m}}
$$

d'où $\quad u M+$ um $=R$.

Les termes uM et um représentent respectivement les contributions de l'engrais et du sol à l'alimentation de la récolte en azote $(\mathrm{R})$. Ainsi, pour une période suffisamment longue, l'azote minéral apparaît utilisé avec le même coefficient, qu'il provienne de l'engrais ou du sol proprement dit (minéralisation).

\section{F. Importance fonctionnelle $d u$ coefficient $\sigma$ dans l'ensemble des transferts de l'azote dans le sol}

Les différents coefficients $\sigma, \omega$ et $u$ relient entre elles des grandeurs extensives relatives à un système sol-plante bien défini. Dans la démarche présentée, $\sigma$ apparaît comme une valeur fondamentale dont dépendent dans une large mesure les dynamiques associées de l'azote et du carbone; en d'autres termes, cela signific que l'ampleur des différents transferts qui affectent le complexe sol-plante est fortement marquée par les caractéristiques métaboliques de la masse microbienne du sol considérée dans son ensemble et pour l'environnement concerné. Cette action dominante de $\sigma$ peut s'expliciter ainsi :

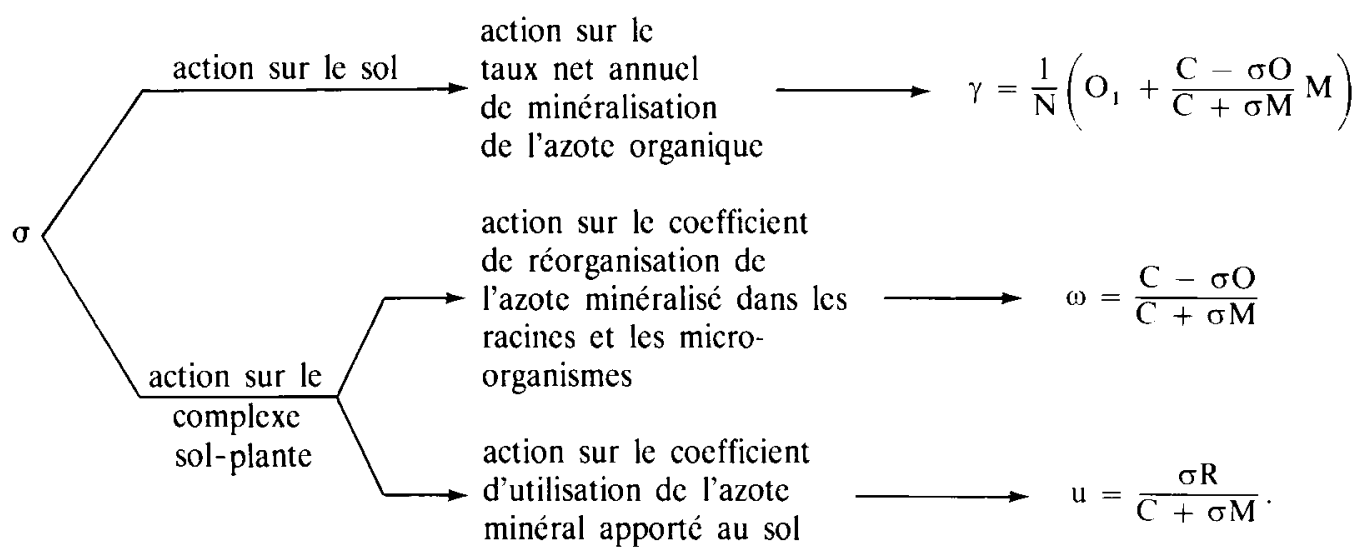


Les lettres $\mathrm{C}, \mathrm{M}, \mathrm{O}, \mathrm{O}_{1}$ et $\mathrm{R}$ ont les significations habituelles. $\gamma$ représente le taux net annuel de minéralisation de l'azote organique du sol. Dans un sol en équilibre organique, $\gamma$ s'explicite sous une forme simple (MOREL, 1971).

$$
\gamma=\frac{\mathrm{O}_{1}+\omega \mathrm{M}}{\overline{\mathrm{N}}}
$$

$\mathrm{N}$ indique la quantité d'azote organique contenue dans la masse de sol considérée qui reçoit respectivement les quantités $\mathrm{O}_{1}$ et $\mathrm{M}$ d'azote organique et d'azote minéral. En remplaçant $\omega$ par sa valeur en fonction de $\sigma, \gamma$ s'exprime par la relation (13).

Dans un sol qui n'a pas atteint son équilibre organique, $\gamma$ se calcule en fonction des quantités $O_{1}, M$ et $\omega$, et des quantités $\mathrm{N}_{0}$ et $\mathrm{N}_{\mathrm{t}}$ d'azote organique du sol aux temps zéro et $t$ (MOREL, 1971).

\section{APPLICATIONS}

\section{A. Matériel expérimental}

Les résultats obtenus dans l'approche théorique proposéc sont appliqués aux données expérimentales recueillies sur un dispositif parcellaire de longue durée implanté sur le Centre de Grignon de l'I.N.A. Paris-Grignon (dispositif DEHÉRAIN).

Maintenus en rotation blé-betterave sucrière depuis 1929 , 7 groupes de parcelles de ce dispositif sont étudiés, recevant les traitements suivants :

1) Sans aucune fumure depuis 1902 .

2) Fumure minérale N.P. K depuis 1902.

3) Fumure minérale N.K sans P depuis 1929.

4) Fumure minérale P. K sans N depuis 1929.

5) Fumure minérale N.P sans K depuis 1929.

6) Fumure au fumier d'étable, sans apport minéral, depuis 1929.

7) Fumure mixte « fumier d'étable $+N . P . K$, depuis 1965.
Les quantités apportées au sol, exprimées en $\mathrm{kg} / \mathrm{ha} / \mathrm{an}$, sont les suivantes

Fumier d'étable

Azote (N) 10000

Phosphore (P)

87 (ammonitrate)

Potassium (K)

40 (superphosphate)

75 (chlorure de potassium)

Les parcelles traitées en fumure mixte reçoivent $10000 \mathrm{~kg}$ de fumier d'étable par ha ainsi qu'une fertilisation complémentaire $\mathrm{N} \cdot \mathrm{P} \cdot \mathrm{K}$ telle que les quantités de $\mathrm{N}, \mathrm{P}$ ct $\mathrm{K}$ incorporées au sol (minérales et organiques) égalent les quantités apportées dans la fertilisation $\mathrm{N} \cdot \mathrm{P} \cdot \mathrm{K}$ (traitement 2). Une analyse annuelle du fumier d'étable utilisé est donc nécessaire.

La quantité d'azote minéral apportée par les pluies est de l'ordre de $10 \mathrm{~kg} / \mathrm{ha} / \mathrm{an}$.

Les résidus aériens des récoltes sont éliminés (paille, verts de betteraves).

\section{B. Méthodes analytiques}

Le dosage du carbone dans le sol est réalisé par oxydation bichromique en milieu sulfurique, à chaud (ANNE, 1945); il est effectué dans les résidus de récoltes par oxydation dans l'oxygène et détermination du $\mathrm{CO}_{2}$ dégagé.

L'azote est dosé dans le sol et les organes végétaux par la méthode de KJELDAHL.

\section{Données expérimentales}

Elles correspondent à l'année moyenne d'une période comprise entre 1938 et 1972 (34 ans).

Si nous exceptons les quantités d'azote minéral apportées au sol, les données présentées dans le tableau 3 se réfèrent aux masses mises en jeu (sol, récoltes) et à leurs teneurs en azote et en carbone.

La production végétale (blé, betterave), déterminée chaque année, permet une estimation des résidus carbonćs restant dans le sol: en ce qui concerne la céréale, les chaumes et autres parties aériennes non récoltées sont évalués à $1 / 5$ du poids des pailles; la masse racinaire est estimée à 30 p. 100 de la somme (grain + paille). Le poids des racines de betteraves, exprimé en matière sèche, est évalué à $6 \mathrm{p}$. $100 \mathrm{du}$ poids de la récolte.

TABLEAU 3

Données expérimentales relatives aux différents groupes de parcelles (exprimées en kg/ha pour une année moyenne). Experimental data concerning different groups of plots ( $\mathrm{kg} / \mathrm{ha} /$ year).

\begin{tabular}{|c|c|c|c|c|c|c|c|c|}
\hline & & $\begin{array}{l}\text { Sans } \\
\text { fumure }\end{array}$ & N.P.K & $\begin{array}{c}\text { N. K } \\
\text { sans P }\end{array}$ & $\begin{array}{c}\text { N. P } \\
\operatorname{sans~K}\end{array}$ & $\begin{array}{c}\text { P. K } \\
\operatorname{sans} N\end{array}$ & $\begin{array}{l}\text { Fumier } \\
\text { d'étable }\end{array}$ & $\begin{array}{l}\text { Fumier } \\
+\mathrm{N} \cdot \mathrm{P} \cdot \mathrm{K}\end{array}$ \\
\hline \multicolumn{9}{|l|}{ Carbone } \\
\hline Apport au sol & $\mathrm{C}_{1}$ & 0 & 0 & 0 & 0 & 0 & 900 & 900 \\
\hline Sortic du compartiment II & $\mathrm{C}_{2}$ & 185 & 154 & 210 & 262 & 287 & 153 & 0 \\
\hline Apport par les racines & $\mathrm{C}_{3}$ & 534 & 1388 & 1059 & 1142 & 803 & 1240 & 1378 \\
\hline Entrées dans le compartiment I & $\mathrm{C}$ & 719 & 1542 & 1269 & 1404 & 1090 & 2293 & 2278 \\
\hline \multicolumn{9}{|l|}{ Azote organique } \\
\hline Apport au sol & $\mathrm{O}_{1}$ & 0 & 0 & 0 & 0 & 0 & 51 & 51 \\
\hline Sortie du compartiment II & $\mathrm{O}_{2}$ & 21 & 18 & 24 & 30 & 33 & 17 & 0 \\
\hline Entrées dans le compartiment I & $\mathrm{O}$ & 21 & 18 & 24 & 30 & 33 & 68 & 51 \\
\hline \multicolumn{9}{|l|}{ Azote minéral } \\
\hline Apport au sol & M & 10 & 97 & 97 & 97 & 10 & 19 & 46 \\
\hline Exportations d'azote par les rćcoltes & $\mathrm{R}$ & 35 & 109 & 85 & 114 & 48 & 85 & 85 \\
\hline Rapport $\mathrm{C} / \mathrm{N} \mathrm{du}$ sol & & 8,7 & 8,7 & 8,8 & 8,7 & 8,8 & 9,1 & 9,3 \\
\hline
\end{tabular}


L'apport de carbone au sol par les résidus de récolte est calculé en tenant compte des teneurs suivantes des différents organes: paille 45 p. 100 de la matière sèche, racines (blé, betterave) 35 p. 100 .

Le taux moyen du fumier en carbone est de 36 p. 100 de la matière sèche ; l'azote ammoniacal, évalué à 15 p. 100 de la teneur en azote total, est compté comme un apport d'azote minéral au sol.

\section{Résultats et commentaires}

Le tableau 4 indique les résultats calculés d'après les données du tableau 1 et pour une valeur $\sigma=24$; cette valeur semble parmi les plus acceptables lorsque, notamment, elle est considérée en fonction du coefficient d'utilisation $\mathrm{u}$ (relation 10).

Plusieurs observations sont suggérées par ces résultats, observations qui, bien entendu, s'inscrivent dans le cadre de la démarche proposée dans ce mémoire :

- Les parcelles « $\mathrm{P} . \mathrm{K} »$, sans fumure azotée, se comportent d'une façon analogue aux parcelles ne recevant aucune fertilisation.

- Le taux de réorganisation est le plus faible lorsque le phosphore ou le potassium sont absents de la fumure (parcelles « $N . K$ » et $« N . P »$ à fertilisation déséquilibrée).

- Les coefficients d'utilisation calculés sont de l'ordre de grandeur de ceux habituellement proposés après des déterminations directes, dans différentes conditions. Ils représentent vraisemblablement les coefficients «cumulés". Le coefficient d'utilisation le plus bas est observé pour les parcelles ne recevant pas de fumure phosphatée $(\mathrm{N} . \mathrm{K})$; il est le plus élevé pour les parcelles sans aucune fumure et sans fertilisation azotée (P.K).

- Le coefficient de pertes, généralement faible pour l'ensemble des parcelles étudiées, est le plus élevé, de façon très nette, pour les parcelles recevant une fumure minćrale sans-phosphore; cette remarque est à relier à la faible valeur du coefficient d'utilisation dans ce traitement.

- Le coefficient de pertes apparaît légèrement négatif pour les parcelles sans aucune fumure et avec une fertilisation sans azote $(P . K)$. Cette anomalie peut s'expliquer par une sous-estimation de la somme $(\mathrm{M}+\mathrm{O})$ des expressions de $b$ et de e (tabl. 2).
Les valeurs de $\mathrm{M}$ et de $\mathrm{O}$ étant faibles, cette sousestimation prend une grande importance relative. La quantité $O$ devrait, plus particulièrement dans ces 2 types de traitements, être majorée de la quantité d'azote organique incorporée directement au sol par fixation non symbiotique : il suffit d'une fixation de $5,3 \mathrm{~kg}$ d'azote $/ \mathrm{ha} / \mathrm{an}$ pour les parcelles « $\mathrm{P}$. K », de $3,7 \mathrm{~kg}$ pour les parcelles sans aucune fumure, pour annuler la valeur négative de II. Tenant compte de ces observations, il est permis de penser que les pertes demeurent très faibles dans ces 2 groupes de parcelles; il semble également opportun, pour l'ensemble des traitements, d'inclure l'apport non symbiotique d'azote organique dans les entrées au compartiment I ; notons à ce propos que les micro-organismes fixateurs font partie de la masse microbienne dont le métabolisme est caractérisé par la valeur $\sigma$. Le tableau 5 reproduit, à titre indicatif, quelques résultats calculés en tenant compte d'une fixation non symbiotique de $6 \mathrm{~kg} / \mathrm{ha} / \mathrm{an}$ pour chacun des traitements étudiés et pour 2 valeurs de $\sigma$ : 24 et 27 .

- Les coefficients $\omega_{1}$ et $\omega_{2}$ indiquant les parts respectives de la réorganisation dans les racines et les micro-organismes, peuvent être calculés dans la mesure où peut être faite une évaluation de $\mathrm{N}_{\mathrm{r}}$, évaluation malheureusement imprécise. Tenant compte du rapport $\mathrm{C} / \mathrm{N}$ des racines de blé et de betterave ainsi que du rapport des masses racinaires de chacune des cultures dans la rotation, il ressort que lc rapport moyen des racines est de l'ordre de 70 ; la quantité $\mathrm{N}_{\mathrm{r}}$ se déduit de la masse $\mathrm{C}_{3}$ de carbone racinaire.

Les valeurs de $\omega_{1}$ et $\omega_{2}$ sont reportées sur le tableau 4 . Le rapport $\omega_{1} / \omega_{2}$ varie de 0,7 à 1,8 pour les parcelles fertilisées ; par contre, la réorganisation dans les racines est largement dominante pour les parcelles sans apport d'azote, observation à rapprocher de la valeur élevée du coefficient d'utilisation pour ces mêmes parcelles.

Notons également la similitude des résultats pour les traitements «N.P.K » et «fumier + N.P.K», ainsi que l'influence d'un apport complémentaire minéral à la fumure au fumier d'étable.

\section{CONCLUSIONS}

L'objet de ce mémoire était de tenter de préciser l'ampleur des processus d'organisation et de réorganisation de l'azote minéral du sol en utilisant des données analyti-

TABLEAU 4

Résultats calculés pour les différents groupes de parcelles $(\sigma=24)$.

Calculated results for the different groups of plots.

\begin{tabular}{|c|c|c|c|c|c|c|c|}
\hline & $\begin{array}{c}\text { Sans } \\
\text { fumure }\end{array}$ & $\mathrm{N} \cdot \mathrm{P} \cdot \mathrm{K}$ & $\begin{array}{l}\mathrm{N} \cdot \mathrm{K} \\
\text { sans } \mathrm{P}\end{array}$ & $\begin{array}{c}\mathrm{N} \cdot \mathrm{P} \\
\operatorname{sans} \mathrm{K}\end{array}$ & $\begin{array}{c}\mathrm{P} \cdot \mathrm{K} \\
\text { sans } \mathrm{N}\end{array}$ & $\begin{array}{l}\text { Fumier } \\
\text { d'étable }\end{array}$ & $\begin{array}{c}\text { Fumier } \\
+\mathrm{N} \cdot \mathrm{P} \cdot \mathrm{K}\end{array}$ \\
\hline A & 30 & 64,2 & 52,9 & 58,5 & 45,4 & 95,5 & 94,9 \\
\hline$\omega$ & 0,22 & 0,29 & 0,19 & 0,18 & 0,23 & 0,24 & 0,31 \\
\hline $\mathrm{u}$ & 0,87 & 0,68 & 0,57 & 0,73 & 0,87 & 0,74 & 0,60 \\
\hline$\prod$ & $-0,093$ & 0,035 & 0,24 & 0,084 & $-0,096$ & 0,015 & 0,085 \\
\hline$\gamma \times 100$ & 0,61 & 1,09 & 0,99 & 1,05 & 0,80 & 1,55 & 1,45 \\
\hline $\mathrm{x}$ & 8,7 & 65,6 & 55,0 & 71,0 & 8,7 & 14,1 & 27,7 \\
\hline$y$ & 26,2 & 43,4 & 30,0 & 42,9 & 39,3 & 70,9 & 57,3 \\
\hline $\mathrm{b}$ & $-0,93$ & 3,4 & 23,2 & 8,1 & $-0,1$ & 0,3 & 3,9 \\
\hline $\mathrm{e}$ & $-2,8$ & 2,2 & 12,6 & 4,9 & $-4,4$ & 1,5 & 8,1 \\
\hline$\omega_{1}$ & 0,19 & 0,12 & 0,10 & 0,10 & 0,21 & 0,15 & 0,14 \\
\hline$\omega_{2}$ & 0,029 & 0,17 & 0,093 & 0,079 & 0,024 & 0,088 & 0,17 \\
\hline$\omega_{1} / \omega_{2}$ & 6,6 & 0,7 & 1,1 & 1,3 & 8,6 & 1,8 & 0,8 \\
\hline
\end{tabular}


TABLEAU 5

Résultats calculés avec de nouvelles données. Calculated results with new data.

\begin{tabular}{|c|c|c|c|c|c|c|c|c|c|}
\hline Traitements & $\sigma$ & A & $\omega$ & $\mathrm{u}$ & $\prod$ & $x$ & $y$ & $\mathrm{~b}$ & $\mathrm{c}$ \\
\hline Sans fumure & 24 & 30,0 & 0,070 & 0,87 & 0,057 & 8,7 & 26,2 & 0,57 & 1,70 \\
\hline \multirow{2}{*}{$N \cdot P \cdot K$} & 24 & 64,2 & 0,25 & 0,68 & 0,072 & 65,6 & 43,4 & 7,0 & 4,6 \\
\hline & 27 & 57,1 & 0,22 & 0,71 & 0,076 & 68,6 & 40,4 & 7,3 & 4,3 \\
\hline \multirow{2}{*}{$\mathrm{N} \cdot \mathrm{K}$ sans $\mathrm{P}$} & 24 & 52,9 & 0,15 & 0,57 & 0,27 & 55,0 & 30,0 & 27,1 & 14,7 \\
\hline & 27 & 47,0 & 0,12 & 0,59 & 0,29 & 57,3 & 27,7 & 28,2 & 13,6 \\
\hline \multirow{2}{*}{$N \cdot P$ sans $K$} & 24 & 58,5 & 0,14 & 0,73 & 0,12 & 71,1 & 42,9 & 11,8 & 7,2 \\
\hline & 27 & 52,0 & 0,11 & 0,76 & 0,13 & 74,2 & 39,8 & 12,4 & 6,6 \\
\hline \multirow{2}{*}{$P \cdot K$ sans $N$} & 24 & 45,4 & 0,12 & 0,87 & 0,012 & 8,7 & 39,3 & 0,12 & 0,53 \\
\hline & 27 & 40,4 & 0,03 & 0,95 & 0,013 & 9,5 & 38,5 & 0,13 & 0,52 \\
\hline \multirow{2}{*}{ Fumier d'étable } & 24 & 95,5 & 0,19 & 0,74 & 0,068 & 14,1 & 70,9 & 1,3 & 6,5 \\
\hline & 27 & 84,9 & 0,11 & 0,82 & 0,075 & 15,5 & 69,5 & 1,4 & 6,3 \\
\hline \multirow{2}{*}{ Fumier $+\mathbf{N} \cdot \mathbf{P} \cdot \mathrm{K}$} & 24 & 94,9 & 0,27 & 0,60 & 0,13 & 27,7 & 57,3 & 5,9 & 12,1 \\
\hline & 27 & 84,4 & 0,21 & 0,65 & 0,14 & 30,0 & 55,0 & 6,3 & 11,7 \\
\hline
\end{tabular}

ques et agronomiques usuelles, le plus souvent en nombre limité, relatives au complexe sol-plante.

Considérée sous cet aspect, toute réflexion sur le problème posé excluait la prise en compte de chaque phénomène biologique élémentaire (minćralisation, nitrification...), très difficile à appréhender dans les conditions naturelles ou agronomiques du développement végétal.

Elle ne pouvait donc se référer qu'à des ensembles ou groupements fonctionnels suffisamment vastes et pour lesquels des hypothèses simples et acceptables permettraient d'en approcher globalement le fonctionnement au plan quantitatif.

Le premier ensemble choisi concerne la fraction "labile» de la matière organique du sol à laquelle nous réservons un rôle fonctionnel dominant.

Le second groupe de référence est représenté par la masse microbienne du sol qui, par la grandeur $\sigma$ qui la caractérise, assume une fonction déterminante dans la distribution de l'azote minéral.

Enfin, pour évaluer l'importance quantitative des différents transferts d'azote, il était nécessaire d'utiliser une règle unique - règle de proportionnalité - précisant le mode de répartition des masses mises en œuvre.

L'application de ces concepts théoriques à des cas concrets conduit à des résultats qui paraissent acceptables et permettent d'apporter des informations relatives au problème étudié dans ce mémoire :
- Au plan général, une approche quantitative des processus de réorganisation de l'azote et, d'une façon complémentaire, une estimation du coefficient d'utilisation par le végétal de l'azote minéral reçu par le sol. Corrélativement, la connaissance du coefficient de réorganisation de l'azote permet l'évaluation du taux net annuel de minéralisation de l'azote organique, grandeur nécessaire, en particulier, au traitement du problème de l'entretien organique des sols.

- Au plan des ouvertures possibles, une analyse plus complète de certains résultats expérimentaux, tels ceux obtenus par des essais parcellaires de longue durée : ainsi, le coefficient d'utilisation de l'azote minéral par la plante augmente lorsque l'azote est déficient dans la fumure ; au contraire, fait assez inattendu, il diminuc dans le cas d'une fertilisation sans phosphore.

Il serait toutefois souhaitable que les propositions ćtablies dans ce mémoirc fussent appliquées à des sols de situations pédo-climatiques et culturales variées. Ces propositions s'appuient par ailleurs sur des obscrvations réalisées à moyen et à long terme; il ne fait aucun doute qu'elles gagneraient à ĉtre confrontées, quant à leurs applications, aux résultats obtenus par des déterminations directes dans la mesure où celles-ci se situeraient à des échelles de temps comparables.

Reçu le 8 février 1983. Accepté le 4 novembre 1983.

\section{RÉFÉRENCES BIBLIOGRAPHIQUES}

Anne P., 1945. Sur le dosage rapide du carbone organique des sols. Ann. Agron., 15, 161-172.

Boniface R., 1981. Le lessivage des nitrates en zone agricole. $4^{e} J$. Sci. et Techn., Paris 1981. Collection Recherche et Environnement, $n^{\circ} 17,91-114$.

Fried M., Dean L. A., 1952. A concept concerning the mcasurement of available soil nutrients. Soil Sci., 73, 263-271.

Lindemann Y., Guiraud J., Christman J., Auge G., 1981. Estimation en plein champ du coefficient d'utilisation par les betteraves sucrières de l'azote minéral apporté. Colloque Int. Humus-Azote, Reims 1981, 174-180.
Morel R., 1971. Evolution dans le temps de la quantité de l'azote organique du sol. Sci. Sol, (1), 121-129.

Morel R., 1981a. Essai d'évaluation de l'organisation et de la réorganisation de l'azote minćral du sol par la méthode des modèles compartimentaux. I. Cas d'un sol maintenu sans culture. Agronomie, 1 (1), 19-26.

Morel R., 1981b. Cinétiques relatives de l'azote et du carbone dans le sol. C.R. Acad. Agric., Fr., 67 (10), 903-910.

Rémy J. C., Viaux Ph., 1980. Le devenir des engrais dans le sol. Perspect. Agric., 43, 5-9. 